\title{
Non-Cell-Autonomous Regulation of GABAergic Neuron Development by Neurotrophins and the p75 Receptor
}

\author{
Pao-Yen Lin, ${ }^{1,2 *}$ Jeanine M. Hinterneder, ${ }^{1 \star}$ Sarah R. Rollor, ${ }^{1}$ and Susan J. Birren ${ }^{1}$ \\ ${ }^{1}$ Department of Biology, Volen Center for Complex Systems, Brandeis University, Waltham, Massachusetts 02454, and ${ }^{2}$ Department of Psychiatry, Chang \\ Gung Memorial Hospital-Kaohsiung Medical Center, Chang Gung University College of Medicine, Kaohsiung, County 831, Taiwan
}

\begin{abstract}
Basal forebrain GABAergic and cholinergic circuits regulate the activity of cholinergic projections to the cortex and hippocampus. Because these projections influence cortical development and function, the development of basal forebrain excitatory and inhibitory neurons is critical for overall brain development. We show that the neurotransmitter phenotype of these neurons is developmentally regulated by neurotrophins and the 75 receptor. Neurotrophins (nerve growth factor and brain-derived neurotrophic factor) increased the number of both cholinergic and GABAergic neurons in neonatal basal forebrain neuron cultures from the region of the medial septum. However, the p75 receptor is required only for neurotrophin-dependent expansion of the GABAergic, not the cholinergic, population. Neurotrophin-induced GABAergic development can be rescued in $p 75^{-/-}$cultures by expression of a p 75 rescue construct in neighboring cells or by treatment with medium collected from neurotrophin-treated wild-type cultures. Because p75 is not expressed in basal forebrain GABAergic neurons, this defines a new, non-cell-autonomous mechanism of p 75 action in which ligand binding results in release of a soluble factor that modifies neurotrophin responses of nearby neurons. p75 is also required for the maintenance of basal forebrain GABAergic neurons in vivo, demonstrating that p75-mediated interactions between cholinergic and GABAergic neurons regulate the balance of excitatory and inhibitory components of basal forebrain circuits.
\end{abstract}

Key words: basal forebrain; neurotransmitter phenotype; neurotrophins; $\mathrm{p} 75$ receptor; cholinergic neurons; GABAergic neurons

\section{Introduction}

Basal forebrain cholinergic neurons send projections to the cortex and hippocampus where they regulate neuronal development and activity (Semba, 2000; Nishimura et al., 2002; Hohmann, 2003). Inhibitory GABAergic neurons in the basal forebrain coproject with cholinergic neurons (Freund and Meskenaite, 1992; Gritti et al., 1997) and exhibit extensive local collaterals onto cholinergic neurons (Zaborszky and Duque, 2000). In turn, cholinergic neurons form reciprocal connections onto the GABAergic neurons (Brauer et al., 1998), defining a local circuit for the regulation of cholinergic output. Thus, mechanisms that control the balance between cholinergic and GABAergic neurons in the basal forebrain may be critical to the development and function of cortical and hippocampal circuitry.

Neurotrophins have long been known to regulate the development of basal forebrain cholinergic neurons (BFCNs). Nerve growth factor (NGF) increases the expression and ac-

\footnotetext{
Received July 20, 2007; revised Sept. 26, 2007; accepted 0ct. 17, 2007.

This work was supported by a grant from the National Alliance for Autism Research (S.J.B.) and by National Institutes of Health/National Institute of Neurological Disorders and Stroke Grant P30 NS45713 for Core Facilities for Neuroscience at Brandeis University. P.-Y.L. was supported by Chang Gung Memorial Hospital, Taiwan, and J.M.H. was supported by National Institutes of Health/National Institute of Neurological Disorders and Stroke Grant T32 NS07292. We thank Dr. Moses V. Chao for 75 constructs, Tatyana Pozharskaya for technical help, Drs. Piali Sengupta and Leslie Griffith for critical reading of this manuscript, and Regeneron Pharmaceuticals for providing BDNF.

*P.-Y.L. and J.M.H. contributed equally to this work.

Correspondence should be addressed to Dr. Susan J. Birren, Department of Biology, Brandeis University, MS 008 , 415 South Street, Waltham, MA 02454. E-mail: birren@brandeis.edu.

D01:10.1523/JNEUROSCI.3302-07.2007

Copyright $\odot 2007$ Society for Neuroscience $\quad 0270-6474 / 07 / 2712787-10 \$ 15.00 / 0$
}

tivity of choline acetyltransferase (ChAT) (Hatanaka et al., 1988) and vesicular acetylcholine transporter (VAChT) (Pongrac and Rylett, 1998; Berse et al., 1999) and increases cholinergic neuron number in vitro (Hatanaka et al., 1988). Brainderived neurotrophic factor (BDNF) enhances cholinergic marker expression and supports survival of postnatal basal forebrain neurons (Nonomura and Hatanaka, 1992; Nonomura et al., 1995; Ward and Hagg, 2000). The effects of neurotrophins on basal forebrain GABAergic neurons are less clear. Although NGF may increase GABAergic neuron numbers in some culture systems (Arimatsu and Miyamoto, 1991), intraventricular infusion in adult rats showed no effects of NGF or BDNF on numbers of GABAergic neurons (Koliatsos et al., 1994). However, target ablation of basal forebrain projections resulted in a partial loss of GABAergic neurons (Plaschke et al., 1997), suggesting a requirement for target-derived factors for GABAergic neuron development or survival.

Although many effects of neurotrophins are mediated through Trk receptors, the lower-affinity p $75^{\text {NTR }}$ receptor (p75) also participates in neurotrophin signaling (Dechant and Barde, 2002). In the basal forebrain, p75 is localized to the cholinergic, but not GABAergic, neurons (Hartikka and Hefti, 1988; Heckers et al., 1994). Studies of the basal forebrain of mice lacking $p 75$ have reported either an increased number of BFCNs (Van der Zee et al., 1996; Yeo et al., 1997; Naumann et al., 2002) or a decrease or no change in number (Hagg et al., 1997; Peterson et al., 1997, 1999; Ward and Hagg, 1999; Greferath et al., 2000). Hence, it is likely that p75 plays a role in regulating basal forebrain neuron 
development, although specific effects on neuronal subpopulations, specifically the GABAergic population, are not clear.

We examined the influence of neurotrophins on the acquisition of cholinergic and GABAergic properties in basal forebrain cultures and investigated the role of the p75 receptor. We demonstrate that neurotrophins promote the expression of both cholinergic and GABAergic neuronal phenotypes and have identified a p75-dependent non-cell-autonomous mechanism for the regulation of GABAergic development in the basal forebrain. These experiments demonstrate a novel role for p75 in establishing the developmental balance of cholinergic and GABAergic neuron numbers.

\section{Materials and Methods}

Dissociated basal forebrain neuron culture. Basal forebrain cultures were prepared from neonatal wild-type or $p 75^{-/-}$mice (Lee et al., 1992) (The Jackson Laboratory, Bar Harbor, ME). Animal protocols were approved by the Brandeis University Institutional Animal Care and Use Committee. The brains were removed and collected in cold artificial CSF (in mM: $126 \mathrm{NaCl}, 3 \mathrm{KCl}, 2 \mathrm{MgSO}_{4}, 1 \mathrm{NaH}_{2} \mathrm{PO}_{4}, 25 \mathrm{NaHCO}_{3}, 10$ dextrose, and 2 $\mathrm{CaCl}_{2}$ ). Meninges surrounding the brains were removed, and the cerebral hemispheres were spread laterally to expose the basal forebrain, in which the septal area (containing basal forebrain neurons from the medial septum (MS) and the horizontal and vertical limbs of the diagonal band of Broca) was dissected as described previously (Hatanaka et al., 1988). Dissected tissues were minced, enzymatically dissociated with papain (Worthington Biochemical, Lakewood, NJ), and triturated with sterile Pasteur pipettes. The resulting cell suspensions were diluted in a culture medium consisting of minimal essential medium (Invitrogen, Carlsbad, CA) supplemented with 5\% fetal bovine serum (Invitrogen), $2 \%$ penicillin/streptomycin (Invitrogen), 2\% B27 supplement (Invitrogen), 1\% L-glutamine (Invitrogen), and $30 \mathrm{~mm}$ dextrose. The neurons were plated $\left(18,000-20,000\right.$ cells $\left./ \mathrm{cm}^{2}\right)$ onto previously established monolayers of cortical astrocytes (see below). In the cultures for transfection experiments, 40,000 cells $/ \mathrm{cm}^{2}$ neurons were plated onto astrocytes. Basal forebrain cultures were incubated in the absence (control condition) or presence of $50 \mathrm{ng} / \mathrm{ml} \mathrm{NGF} \mathrm{(Upstate} \mathrm{Biotechnology,} \mathrm{Lake}$ Placid, NY) and $50 \mathrm{ng} / \mathrm{ml} \mathrm{BDNF}$ (gift from Regeneron, Tarrytown, NY) during the entire culture period and maintained in a $37^{\circ} \mathrm{C} / 5 \% \mathrm{CO}_{2}$ incubator. One day after plating the cells, $1 \mu \mathrm{M}$ cytosine arabinofuranoside (AraC) (Sigma, St. Louis, MO) was added to the cultures to reduce nonneuronal cell division. Half of the culture medium was replaced every 2-3 d.

Astrocyte cultures were prepared by plating postnatal day $0-3$ mouse visual cortical cells $\left(20,000\right.$ cells $\left./ \mathrm{cm}^{2}\right)$ in the same medium as used for culturing basal forebrain neurons but excluding the B27 supplement. The cultures became a confluent monolayer by $10 \mathrm{~d}$, at which time neurons were plated. Cultured astrocytes have been reported to express neurotrophins (Rudge et al., 1996); thus, these cultures may contain low levels of endogenous neurotrophins that could vary with the final glial density obtained in different experiments.

Conditioned medium (CM) was collected from basal forebrain cultures prepared from wild-type or $p 75^{-/-}$mice, with or without NGF and BDNF ( $50 \mathrm{ng} / \mathrm{ml}$ each) treatment. Half of the culture medium was collected every $2 \mathrm{~d}$, filtered through a $0.45 \mu \mathrm{m}$ filter (Corning, Corning, $\mathrm{NY}$ ), and stored at $-80^{\circ} \mathrm{C}$.

Immunocytochemistry. Basal forebrain cultures were fixed in $4 \%$ paraformaldehyde 2, 6, or $10 \mathrm{~d}$ after plating. Cells were permeabilized with $0.1 \%$ Nonidet P-40 (Sigma) in PBS for 10 min and blocked in 5\% horse serum for $1 \mathrm{~h}$ at room temperature. Cells were incubated at $4^{\circ} \mathrm{C}$ overnight in primary antibody diluted in $1 \%$ horse serum/PBS. Primary antibodies used were mouse anti-microtubule-associated protein 2 (MAP2) (1:2000; Sigma) or chicken anti-MAP2 (1:2000; Chemicon, Temecula, CA), rabbit anti-glutamic acid decarboxylase (GAD) 65/67 (1:2000; Chemicon), mouse anti-GAD67 (1:2000; Chemicon), goat anti-VAChT (1:4000; Chemicon), rabbit anti-active caspase 3 (1:100; Chemicon), rabbit anti-bromodeoxyuridine (BrdU) (1:800; Megabase, Lincoln, NE), and rabbit anti-human p75 receptor intracellular epitope (1:500; Pro- mega, Madison, WI). Primary antibodies were detected after incubation with AMCA (aminomethylcoumarin acetate), FITC-, rhodamine-, or cyanine 5 (Cy5)-conjugated secondary antibodies (1:200; Jackson ImmunoResearch, West Grove, PA) for $1 \mathrm{~h}$ at room temperature.

Stained cells were viewed using an Olympus Optical (Center Valley, PA) IX-70 inverted fluorescent microscope fitted with UV, FITC, rhodamine, and Cy5 filters. Images were captured using an Orca-ER CCD digital camera (Hamamatsu, Shizuoka, Japan) and Openlab software (version 4.0.2; Improvision, Lexington, MA).

To evaluate cell proliferation, $1 \mathrm{~mm}$ BrdU (Roche Diagnostics, Mannheim, Germany) was added for the last $8 \mathrm{~h}$ of the culture period. AraC was not added to these cultures. Cells were fixed as described, washed, treated with $2 \mathrm{~N} \mathrm{HCl}$ in $\mathrm{PBS}$ for $10 \mathrm{~min}$ at room temperature, washed, and treated with $0.1 \mathrm{M} \mathrm{Na}_{2} \mathrm{~B}_{4} \mathrm{O}_{7} \cdot 10 \mathrm{H}_{2} \mathrm{O}$ for $10 \mathrm{~min}$ at room temperature. Cultures were immunostained as described above.

In the cultures with double staining of GAD/MAP2 and VAChT/ MAP2, the percentage of GABAergic and cholinergic neurons was determined by counting the numbers of GAD-positive $\left(\mathrm{GAD}^{+}\right)$or $\mathrm{VAChT}^{+}$ cells, divided by the numbers of MAP2 ${ }^{+}$cells, in the central strip of each culture well. When counting the cells, researchers were blind to the conditions of the experiment. The resulting cell counts were limited by the intensity of the fluorescence staining, the sensitivity of the viewer's eyes, and the optics of the microscope. Duplicate wells for each condition were counted for a minimum of three experiments per condition. For control purposes, each experiment included the culture processed in the absence of the primary antibody.

For the measurement of soma sizes, images of double-stained GAD ${ }^{+}$ neurons and their corresponding MAP2 stain or triple-stained $\mathrm{VAChT}^{+}$, $\mathrm{MAP}^{+}{ }^{+}$, and $\mathrm{p} 75^{+}$neurons were taken using a $40 \times$ objective with 0.85 numerical aperture. Using NIH ImageJ software, neuronal cell bodies were outlined by hand for the MAP2 stain with the freehand selection tool and measured for area.

Western blots. Lysates were isolated from 2 and $10 \mathrm{~d}$ cultures, and protein was assayed using the Bio-Rad (Hercules, CA) Protein Assay with a BSA standard. Fifteen micrograms of protein and molecular weight standards were run on $7.5 \%$ separating gels and electroblotted to nitrocellulose membranes (Schleicher \& Schuell, Keene, NH). The membranes were probed with primary antibodies against GAD (1:7000; Chemicon) or $\beta$-actin (1:15,000; Sigma) for $2 \mathrm{~h}$ at room temperature. After washing, the blots were incubated in HRP-conjugated secondary antibodies (1:10,000; Jackson ImmunoResearch) for $1 \mathrm{~h}$. Immune complexes were visualized by Western Lightning Chemiluminescence Reagent Plus (PerkinElmer, Boston, MA) and exposed to film. The densities on the film of $\sim 42 \mathrm{kDa}$ ( $\beta$-actin) and $65 \mathrm{kDa}$ (GAD65) immunoreactive bands were quantified with background subtraction using Quantity One software (Bio-Rad). All data are obtained as arbitrary units normalized to $\beta$-actin.

Gene transfection. Human p75 was expressed in basal forebrain cultures prepared from $p 75^{-/-}$mice using Lipofectamine 2000 reagent (Invitrogen). Cells were transfected $1 \mathrm{~d}$ after plating with pEGFP-C1 (control; Clontech, Palo Alto, CA), with pEGFP-C1 and pCMV5A, an expression vector containing a human $\mathrm{p} 75 \mathrm{cDNA}$, or with pEGFP-C1 and p75-105 (Yan and Chao, 1991), encoding a p75 protein deficient in ligand binding ( 75 constructs were gifts from Dr. Moses V. Chao, New York University School of Medicine, New York, NY). Expression of p75 was detected by staining with an anti-p75 antibody (Promega) that recognized the intracellular domain of human $\mathrm{p} 75$ protein.

Brain slice preparation, imaging, and analyses. Adult wild-type (C57BL/ 6J) and $p 75^{-/-}$mice (Lee et al., 1992) at 3 or 10-14 months of age were anesthetized (with chloroform and then injected with ketamine, xylazine, and acepromazine mix) and killed by transcardiac perfusion with saline and $4 \%$ paraformaldehyde in phosphate buffer, $\mathrm{pH}$ 7.4. Brains were rapidly dissected out, postfixed overnight at $4^{\circ} \mathrm{C}$, and preserved in $30 \%$ sucrose at $4^{\circ} \mathrm{C}$. Coronal slices ( $40 \mu \mathrm{m}$ thick) containing the MS were made on a vibratome and collected in PBS, beginning where the corpus callosum first begins to cross until the anterior commissure crosses. Alternating slices were immunostained for cholinergic and GABAergic neurons using a free-floating procedure. Sections were blocked and permeabilized in solution containing $0.1 \%$ NP-40 with $10 \%$ donkey serum 
in PBS for $30 \mathrm{~min}$ at room temperature on a shaker at low setting. They were then incubated overnight in the same solution plus primary antibody. After washing three times in PBS (10 min each), the primaries were visualized using FITC- (or Cy2-) and Cy5-conjugated secondary antibodies (Jackson ImmunoResearch). For GABAergic neuron stains, the antibodies used were rabbit anti-GABA (1:4000; Sigma) and mouse antineuronal-specific nuclear protein (NeuN) (1:1000; Chemicon) for quantifying total neuron numbers. To identify cholinergic neurons, a goat polyclonal antibody to ChAT was used (1:500, AB144P; Chemicon). To assess costaining of p75 and cholinergic markers, slices were costained for ChAT and p75 (rabbit polyclonal against human p75, 1:500; Promega). Slices were washed three to four times in PBS, mounted on Superfrost plus slides in $n$-propyl gallate, and stored at $4^{\circ} \mathrm{C}$ until imaged.

The medial septal region of each brain slice was imaged using the $10 \times$ objective on a Leica (Nussloch, Germany) SP2 confocal microscope, taking a series (or stack) of images simultaneously for GABA or ChAT and NeuN stains at $2 \mu \mathrm{m}$ intervals through the slice. These image series were then analyzed using NIH ImageJ software. Image stacks were $z$-projected into one image for each stain using the maximum intensity values for each stack to allow for viewing all cell somas through the slice in one image. The region of the MS was defined and outlined by hand using the freehand tool for the NeuN image and then copied onto the corresponding GABA or ChAT image. The MS region is visually apparent in the NeuN stain from surrounding tissue by its specific cell density and morphology. (The neuronal somas appear more clustered together and have a common directionality.) The total MS area was measured in pixels and converted to square micrometers. Then, the total number of neurons $\left(\mathrm{NeuN}^{+}\right.$cells) and the number of either GABAergic $\left(\mathrm{GABA}^{+}\right)$or cholinergic $\left(\mathrm{ChAT}^{+}\right)$cells within each MS were counted by hand using the Pointpicker function and identifying positive cells by eye while blinded to condition. The number of GABAergic and cholinergic neurons per total number of cells within the region was then computed and averaged across slice and then across mice. In addition, each brain slice was viewed under low magnification and assigned an anatomical coordinate value as "distance from bregma" based on anatomical landmarks present in the slice and by comparison to slices in the mouse brain atlas (Paxinos and Franklin, 2001). These coordinates were then used to further examine if differences in ratios of cholinergic and GABAergic neurons change along the anteroposterior (rostrocaudal) axis of the MS.

An inverted Olympus Optical IX-81 scope equipped with epifluorescence and automatic focus drive was used to image cortical areas of three wild-type and three $p 75^{-1-}$ brains used previously for imaging the MS. A $438.55 \times 334.13 \mu \mathrm{m}$ region of the somatosensory cortex was imaged at 2 $\mu \mathrm{m}$ intervals using Volocity software (Improvision). These image series were then analyzed using NIH ImageJ to quantify the ratio of GABAergic neurons in the cortex in the same manor as described above for the MS.

Statistics. Significance was analyzed by Student's $t$ tests or ANOVA followed by post hoc tests using StatView software (Abacus Concepts, Berkeley, CA).

\section{Results \\ Neurotrophins increase the number of cholinergic and GABAergic neurons}

We examined the regulation of anterior basal forebrain cholinergic and GABAergic neuron numbers by neurotrophins using a well defined in vitro system (Hartikka and Hefti, 1988; Ha et al., 1999; Lopez-Coviella et al., 2005) in which neurons from the neonatal medial septum and diagonal bands of Broca were dissociated and cultured on astrocyte monolayers. We used immunolabeling for VAChT to identify cholinergic neurons and GAD65/67 to label GABAergic neurons (Fig. 1A). Cultures were treated with or without $50 \mathrm{ng} / \mathrm{ml}$ of both NGF and BDNF to examine overall population effects of neurotrophins. Treatment was for 2, 6, and $10 \mathrm{~d}$ followed by immunostaining. Labeling for MAP2 was used to identify all neurons in the cultures. In the absence of added neurotrophins, $\sim 30 \%$ of the neurons expressed VAChT, a number that did not change significantly over the culture period (Fig. 1 B). At $2 \mathrm{~d}, \sim 20 \%$ of the neurons expressed a GABAergic phenotype, increasing to $>30 \%$ by $6 \mathrm{~d}$ in culture (Fig. 1C). The percentage of cholinergic neurons was significantly increased by neurotrophin treatment (Fig. $1 B$ ), as was the percentage of GABAergic neurons (Fig. 1C). In addition, neurotrophins significantly increased soma size for both cholinergic and GABAergic neurons (Fig. 1D). Control cultures without primary antibody did not show immunoreactivity (data not shown). There was no significant change in the total number of MAP2immunopositive cells with neurotrophin treatment (data not shown).

Neurotrophin-dependent increases in cholinergic and GABAergic neurons could be a consequence of increased proliferation or neuronal survival, or recruitment of neurons to a cholinergic or GABAergic phenotype. Birthdating of rat BFCNs has shown that developing neurons withdraw from the cell cycle by embryonic day 17 (E17) (Semba and Fibiger, 1988; Brady et al., 1989), suggesting that neuronal proliferation in our neonatal cultures is unlikely to underlie the effects of neurotrophins. We tested whether a neurotrophin-responsive proliferative cell population could give rise to neurons by pulsing neurotrophintreated and untreated cultures with BrdU for $6 \mathrm{~h}$ before fixation. We found that none of the $\mathrm{MAP}^{+}{ }^{+}$neurons were BrdU labeled (Table 1), demonstrating that neurotrophin-dependent increases did not result from proliferation. We also examined whether neurotrophins promoted the preferential survival of young cholinergic or GABAergic neurons. Cultures were labeled for active caspase 3, a marker of apoptosis, after a $12 \mathrm{~h}, 2 \mathrm{~d}$, or $6 \mathrm{~d}$ treatment with neurotrophins. Fewer than $2.5 \%$ of the neurons were immunolabeled for caspase 3 at any time point (Table 1), and the percentage was not affected by neurotrophins. The absence of proliferative or survival effects suggests that neurotrophins promote the expression of a cholinergic or GABAergic phenotype in VAChT-negative/GAD-negative neurons.

\section{Neurotrophin response of GABAergic neurons requires p75}

Basal forebrain neurons maintain expression of the p75 neurotrophin receptor through postnatal development. We investigated whether p 75 regulates neurotransmitter phenotypes in cultured basal forebrain neurons from the medial septal region. We prepared cultures from neonatal mice deficient in the $\mathrm{p} 75$ receptor $\left(p 75^{-/-}\right)$(Lee et al., 1992) and quantified the number of cholinergic and GABAergic neurons when treated with or without NGF and BDNF. Neurotrophins significantly increased the percentage of cholinergic neurons at both 2 and $6 \mathrm{~d}$ (Fig. 2A), indicating that $\mathrm{p} 75$ was not required for the neurotrophin effect on cholinergic expression. In contrast, we found no neurotrophindependent increase in GABAergic neuron number in $p 75^{-/-} \mathrm{cul}-$ tures (Fig. $2 B$ ). The number of GABAergic neurons in both neurotrophin-treated and untreated cultures increased between 2 and $6 \mathrm{~d}$, reaching a maximal number that was similar to neurotrophin-treated wild-type neurons. This increase was not dependent on the presence of neurotrophins, suggesting that p75 plays a role in limiting GABAergic neuron number in the absence of neurotrophins. A comparison with neurotrophin responses in wild-type cultures (Fig. $2 C, D$ ) shows that p75 is necessary for neurotrophin-dependent increases in GABAergic but not cholinergic neurons.

Although p75 is necessary for neurotrophin-dependent increases in GABAergic neuron number, not all neurotrophin responses require p75. GABAergic soma size was increased after neurotrophin treatment in both wild-type (Fig. $1 D$ ) and $p 75^{-/-}$ (Fig. $3 A$ ) cultures. In addition, Western blot analysis revealed 
neurotrophin-dependent regulation of GAD65 protein levels (Fig. 3B). These results indicate that $\mathrm{p} 75$ is selectively required for neurotrophin-dependent recruitment of neurons to a GABAergic neurotransmitter phenotype but is not required for other aspects of their development, including the regulation of GAD expression in neurons already expressing a GABAergic phenotype.

\section{Expression of p75 in}

cholinergic neurons

The requirement for p75 in establishing the GABAergic phenotype was surprising because p75 expression has been reported to be restricted to the cholinergic population (Hartikka and Hefti, 1988; Heckers et al., 1994). In contrast, Trk receptors are expressed by both GABAergic and cholinergic neurons (Kordower et al., 1994). We therefore examined the expression of p75 in cholinergic and GABAergic neurons in our neonatal cultures. Cultures were quadruple labeled with VAChT and GAD to identify cholinergic and GABAergic neurons, respectively, with p75 to identify neurotransmitter phenotypes coexpressing the neurotrophin receptor and with MAP2 to mark all neurons in the culture (Fig. 4A). We found that p75 was exclusively expressed in the VAChT-expressing population in vitro. We also examined the expression of p75 in cholinergic neurons in vivo. Coronal sections through adult mouse medial septum were stained for ChAT and p75 (Fig. 4B). We found that p75 was exclusively expressed in the ChAT-expressing cholinergic neurons. It appeared that all ChAT-expressing neurons in the medial septum coexpressed p75, although expression levels of p75 were somewhat variable. This expression pattern is specific to the cholinergic neurons of the basal forebrain in that ChATexpressing neurons in other brain regions such as the striatum did not coexpress p75 (data not shown).

\section{p75 promotes neurotrophin-dependent GABAergic development}

The p75 expression pattern in basal forebrain cultures and in vivo raised the possibility that p75 acted in a non-cell-autonomous manner to regulate neurotrophin-dependent acquisition of a GABAergic phenotype. To explore this possibility, we overexpressed $\mathrm{p} 75$ in individual basal forebrain neurons prepared from $p 75^{-/-}$mice and examined the neurotransmitter phenotype of surrounding neurons. We transfected cultures with green fluorescent protein (GFP) or GFP and human p75 $1 \mathrm{~d}$ after plating the neurons. In addition, some neurons were transfected with GFP and p75-105, a 75 mutant that lacks a ligand binding component (Yan and Chao, 1991). In pilot experiments, we found that $94 \%$ of neurons trans-
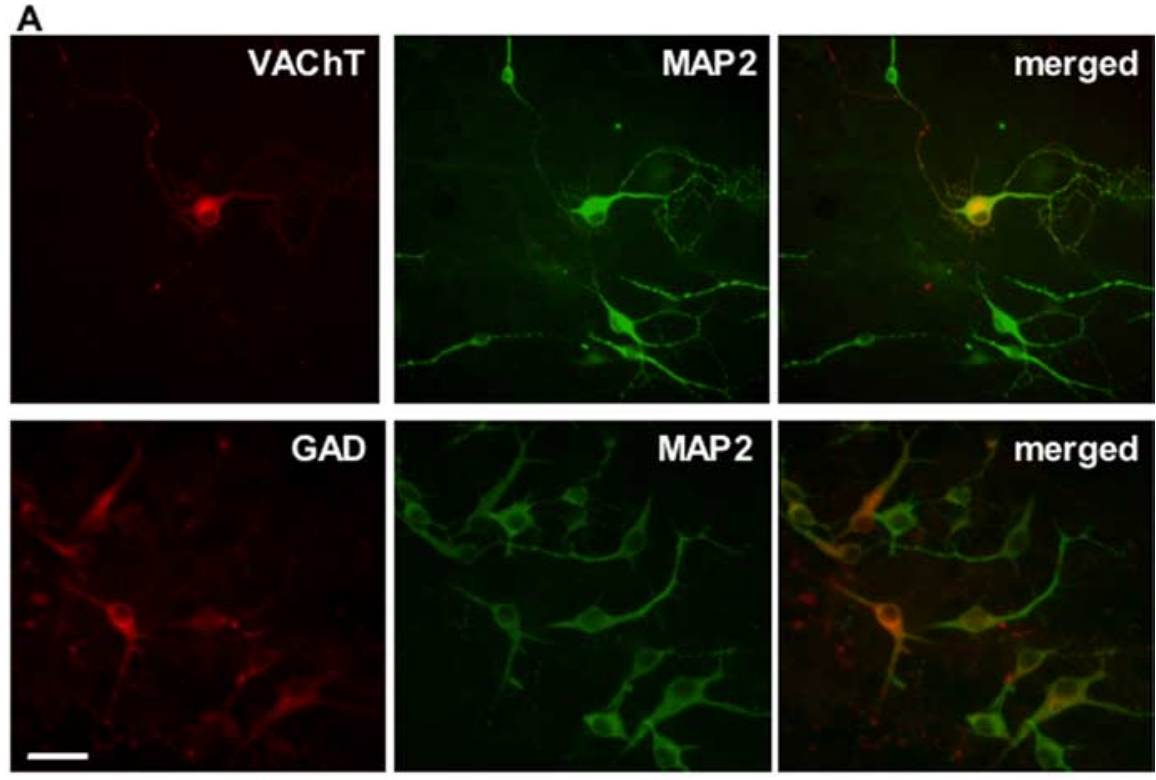

B

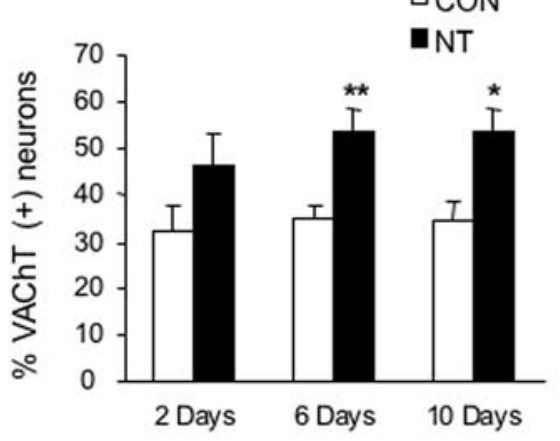

Neuron soma area

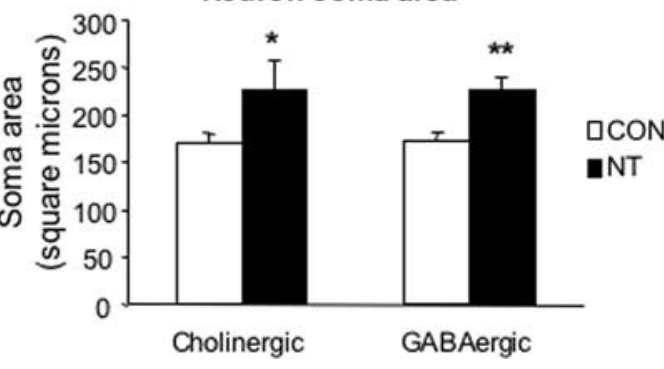

Figure 1. Neurotrophins promote the development of cholinergic and GABAergic neurons in basal forebrain cultures. Basal forebrain neurons were immunostained for MAP2 (green) and for GAD or VAChT (red) markers for GABAergic or cholinergic neurons, respectively. $A$, Double-stained images show a subset of basal forebrain neurons that are GABAergic or cholinergic. Scale bar, $30 \mu \mathrm{m}$. $\boldsymbol{B}, \boldsymbol{C}$, After 2, 6, and $10 \mathrm{~d}$ in cultures in control (CON, open bars) or $50 \mathrm{ng} / \mathrm{ml}$ NGF and BDNF (NT, filled bars) the percentage of $\mathrm{VAChT}^{+}(\boldsymbol{B})$ or $\mathrm{GAD}^{+}(\boldsymbol{C})$ cells that colabeled with MAP2 was measured. Values are given as mean percentage \pm SEM of the number of $\mathrm{VAChT}^{+}$or $\mathrm{GAD}^{+}$cells/the number of MAP2 ${ }^{+}$cells (at least 4 independent experiments in each condition). $D$, The soma area of $\mathrm{VAChT}^{+}$and GAD ${ }^{+}$neurons was measured at $2 \mathrm{~d}$ in cultures $(n=4$ experiments for cholinergic neurons and $n=3$ for GABAergic neurons). ${ }^{*} p<0.05,{ }^{* *} p<0.01$, unpaired $t$ test. fected with two constructs coexpressed both markers, indicating that GFP-positive cotransfected neurons were likely to be p75 positive (Fig. $5 A$ and data not shown). Transfected neurons were cultured in the presence or absence of neurotrophins and, after a $7 \mathrm{~d}$ culture period, the number of $\mathrm{GAD}^{+}$neurons within a $300-\mu \mathrm{m}$-diameter circle around the transfected neuron was determined (Fig. $5 B$ ). The cultures were double labeled with MAP2 to determine the percentage of GABAergic 
Table 1. Neurotrophin effects on cell proliferation and death in basal forebrain cultures

\begin{tabular}{lll}
\hline & CON & NT \\
\hline $\begin{array}{l}\text { Percentage of } \text { BrdU }^{+} \text {neurons } \\
2 \mathrm{~d}(n=3)\end{array}$ & 0 & \\
$\begin{array}{l}\text { Percentage of active caspase } 3^{+} \text {neurons } \\
12 \mathrm{~h}(n=3)\end{array}$ & $0.7 \pm 0.2$ & \\
$2 \mathrm{~d}(n=3)$ & $1.4 \pm 0.6$ & $0.4 \pm 0.1$ \\
$6 \mathrm{~d}(n=4)$ & $2.3 \pm 0.5$ & $2.4 \pm 0.4$ \\
\hline
\end{tabular}

Percentage of $\mathrm{MAP2}^{+}$cells that costained with BrdU or active caspase 3 in basal forebrain cultures treated with (NT) or without (CON) neurotrophins. Values are given as mean \pm SEM. For each experiment, two wells of cultures, each containing at least $600 \mathrm{MAP2}^{+}$cells, were analyzed.

\section{A}

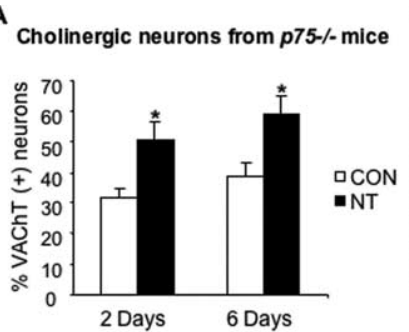

B

GABAergic neurons from $p 75-/-$ mice

C
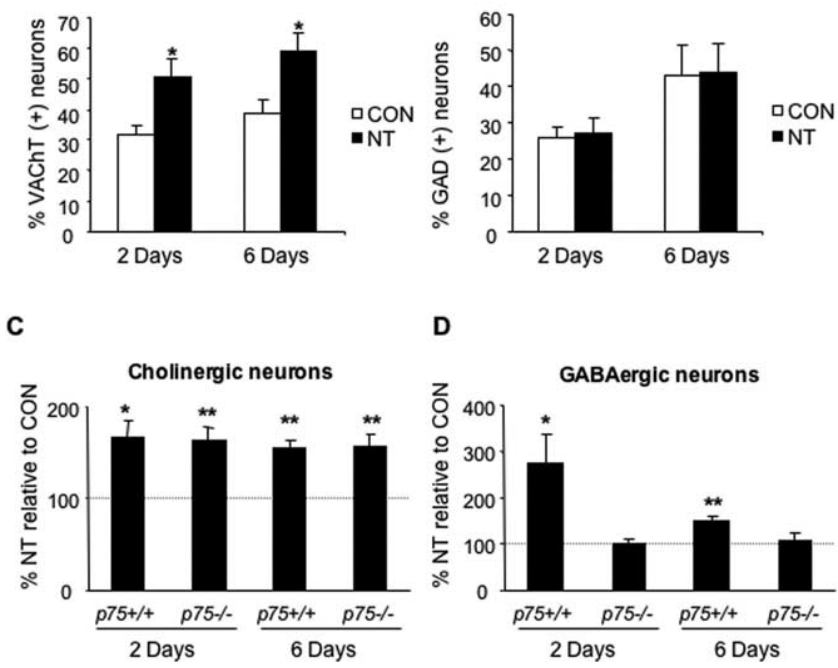

D

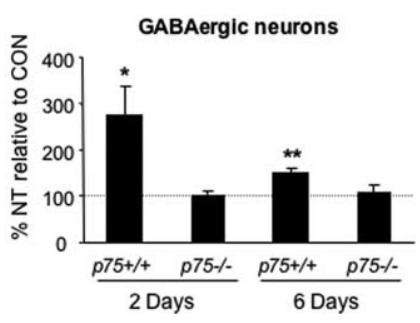

Figure 2. $\quad$ p75 is required for GABAergic, but not cholinergic, neurotrophin responses. Basal forebrain neurons from $p 75^{-/-}$mice were cultured with (NT, filled bars) or without (CON, open bars) neurotrophins. The percentage of $\mathrm{VAChT}^{+}(\boldsymbol{A})$ or $\mathrm{GAD}^{+}(\boldsymbol{B})$ cells that colabeled with MAP2 was measured (values are mean $\pm S E M ; n=$ a minimum of 6 independent experiments), ${ }^{*} p<0.05$, unpaired $t$ test. C, A comparison of wild-type $\left(p 75^{+/+}\right.$) and $p 75^{-/-}$ cultures shows that neurotrophins significantly increase cholinergic neurons in both cultures. $\boldsymbol{D}$, Neurotrophins increase GABAergic neuron percentage in $p 75^{+/+}$, but not $p 75^{-/-}$, cultures (values are mean \pm SEM percentage of NT relative to CON condition). ${ }^{*} p<0.05,{ }^{* *} p<0.01$, unpaired $t$ test.

neurons in close proximity to $p 75$ or control transfected neurons. There was an average of $16.5 \pm 4.7 \mathrm{MAP} 2^{+}$cells in individual circles. Neurotrophin treatment significantly increased the percentage of GABAergic neurons in the proximity of p75-overexpressing neurons (Fig. 5C, GFP $+p 75)$. In contrast, there was no neurotrophin-dependent increase in GABAergic neurons near neurons expressing GFP alone or the ligand binding-deficient $p 75$ variant (Fig. 5C, GFP + p75-105). p75-105expressing neurons did not confer neurotrophin responsiveness on nearby neurons, although in the absence of added neurotrophins there was a small, but not significant, increase in the percentage of GABAergic neurons that may reflect a ligand-independent activity of this variant (Yang et al., 2002). We also measured the percentage of cholinergic neurons in the circled area surrounding transfected neurons. There was a significant increase in cholinergic neuron percentage in neurotrophintreated cultures in the proximity of each type of transfected neuron (Fig. $5 D)$. These results confirm that p75 is not required for the neurotrophin-dependent acquisition of a cholinergic phenotype by basal forebrain neurons and demonstrate a non-cell-autonomous effect of 75 in the regulation of GABAergic properties.
A

\section{Soma area of GABAergic neurons}

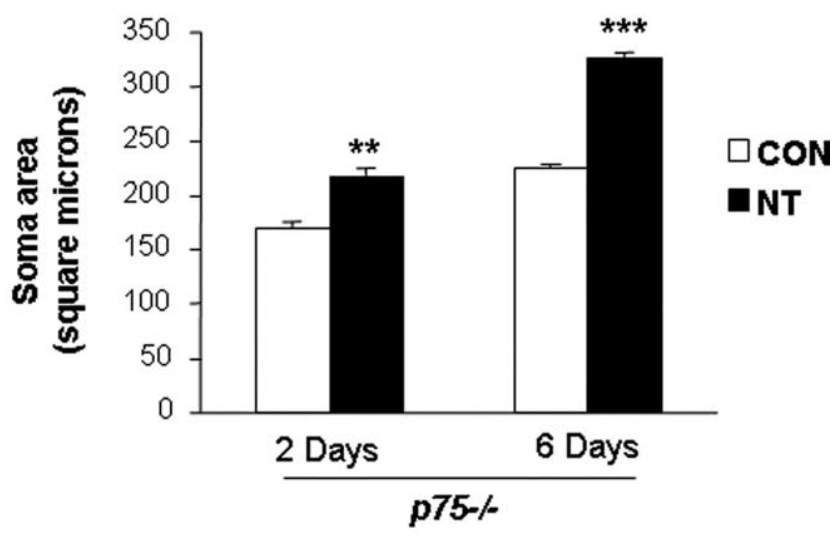

\section{B GAD65 protein level}

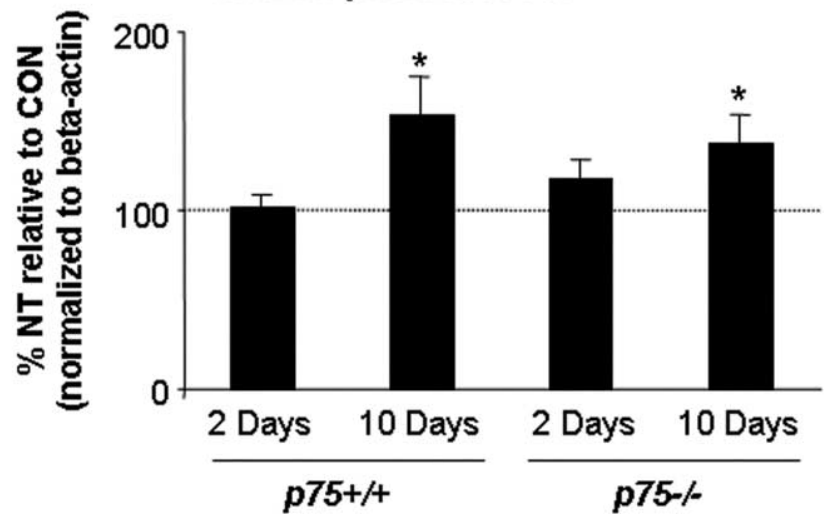

Figure 3. GABAergic neurotrophin responses in the absence of $p 75 . \boldsymbol{A}$, Soma area of $G A D^{+}$ neurons derived from $p 75^{-/-}$cultures. Neurotrophin treatment (NT) is significantly different from the control (CON) at both 2 and 6 d in cultures (mean \pm SEM; $n=3$ ). ${ }^{* *} p<0.01,{ }^{* * *} p<$ 0.001 , unpaired $t$ test. $\boldsymbol{B}$, Western analysis of GAD65 protein in basal forebrain cultures from $p 75^{+1+}$ and $p 75^{-/-}$mice. Neurotrophin treatment (NT) increased GAD65 protein levels at $10 \mathrm{~d}$ in cultures in both $p 758^{+/+}$and $p 75^{-/-}$conditions compared with control (CON). The protein level was normalized to $\beta$-actin. Values are given as mean \pm SEM percentage of intensity of GAD65 band intensity of NT relative to CON condition $(n=6) .{ }^{*} p<0.05$, unpaired $t$ test.

p75 mediates neurotrophin-dependent GABAergic development via secreted factors

The non-cell-autonomous effects of p75 could be mediated through cell-cell interactions, sequestration or presentation of local factors, or the p75-mediated, neurotrophin-dependent release of soluble factors by cholinergic neurons. We distinguished these possibilities by treating $p 75^{-/-}$basal forebrain neurons with conditioned medium generated from neurotrophin-treated wild-type cultures (CM-WT/NT). We found that CM-WT/NT was sufficient to restore the neurotrophin-dependent increase in GABAergic neurons in $p 75^{-/-}$cultures (Fig. $6 A$ ). Residual neurotrophins in the CM-WT/NT apparently had reduced activity after storage, freezing, and thawing of the conditioned medium (see Materials and Methods) because fresh neurotrophins added to the CM-WT/NT-treated $p 75^{-1-}$ cultures resulted in a significant increase in GABAergic neuron number.

Conditioned medium obtained from $p 75^{-/-}$cultures (CM$\mathrm{p} 75 \mathrm{KO} / \mathrm{NT}$ ) did not restore neurotrophin effects on GABAergic neuron number (Fig. $6 \mathrm{~B}$ ), indicating that p75 is required for the production of the conditioned medium factor(s). In addition to 775 
expression, added neurotrophins are also required for production of the conditioned medium activity. Conditioned medium generated in wild-type cultures in the absence of NGF and BDNF did not restore the GABAergic neurotrophin response to $p 75^{-/-}$cultures (Fig. $6 B$ ). These data suggest that neurotrophins act on p75-expressing cells to induce a soluble activity that permits neighboring, p75-negative neurons to respond to neurotrophins by expressing a GABAergic phenotype.

\section{p75 promotes neurotrophin-dependent} GABAergic development in vivo

We have shown that p75 expression in the medial septum is restricted to cholinergic neurons both in vitro and in vivo (Fig. 4). We therefore asked whether p75 has a non-cell-autonomous effect on GABAergic development in the animal by examining GABAergic neuron number in the medial septum of $p 75^{-/-}$mice. We analyzed brain slices through the medial septum from adult wild-type and $p 75^{-/-}$mice (3 or 10-14 months of age; see Materials and Methods). Slices were immunostained for GABA or ChAT and the neuronal nuclear marker NeuN to label the GABAergic neurons, cholinergic neurons, and total population of neurons. Slices were imaged on a confocal microscope, and the medial septal region was outlined based on images of the NeuN stain. GABAergic neurons and total neurons were counted in the identified region, and the ratio of GABAergic neurons to total neurons was determined. The proportion of GABAergic neurons was lower in $p 75^{-/-}$mice compared with wild-type age-matched 10- to 12-monthold animals (Fig. 7A) but not in 3-monthold mice (data not shown). There was no significant difference in total neuron density between the wild-type and $p 75^{-/-}$ mice (Fig. 7B).

We also asked whether the decrease in GABAergic neurons in the $p 75^{-1-}$ mice was generalized in different brain regions by examining a region of the somatosensory cortex that does not receive projections from the medial septum and was contained within the same slices that were imaged for the medial septum. In adult animals, the neurons in this region did not express p75 (data not shown). There was no difference in GABAergic neurons in somatosensory cortex between agematched 10 - to 12 -month-old $p 75^{-/-}$and wild-type mice (Fig. 7C), consistent with the idea that p75-expressing cholinergic neurons play a role in setting the number of GABAergic neurons in the medial septal region.

Finally, we examined whether there were differences in medial septal cholinergic neurons in the $p 75^{-/-}$and wild-type mice. Initial analysis showed a trend toward increased cholinergic neu-ron ratios in the 10 - to 14 -month-old $p 75^{-/-}$ani-
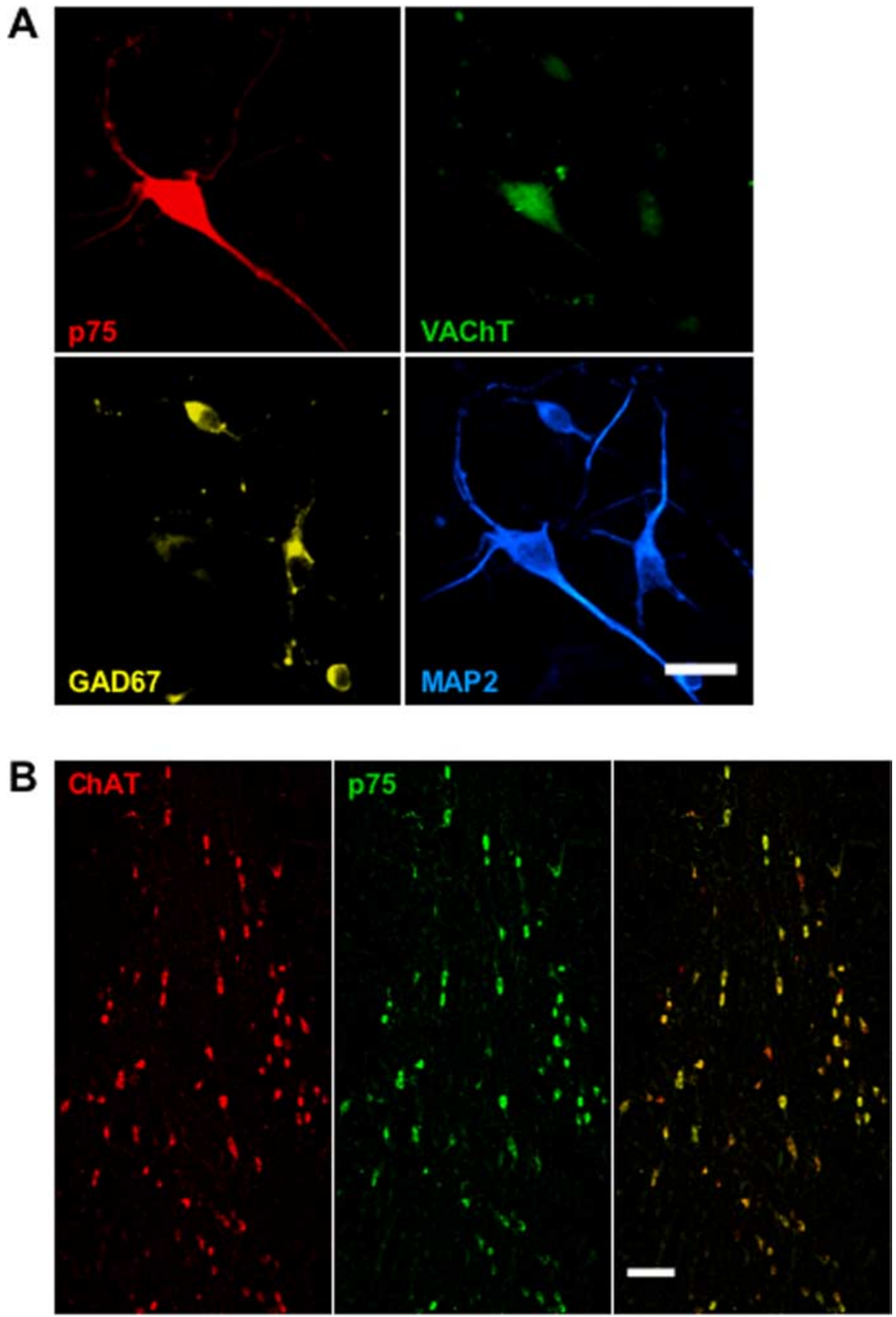

Figure 4. p75 is expressed by cholinergic neurons. Cholinergic neurons from the medial septal region of the basal forebrain exclusively express p75. $\boldsymbol{A}$, Cultures treated with neurotrophins were fixed at $6 \mathrm{~d}$ and quadruple immunostained for p 75 (red), VAChT (green), GAD67 (yellow), and MAP2 (blue). In this image set, there is one $75^{+} / \mathrm{NAChT}^{+}$cholinergic neuron surrounded by non-p75-expressing, GAD67 ${ }^{+}$GABAergic neurons. Scale bar, $25 \mu \mathrm{m}$. B, Coronal brain sections (40 $\mu \mathrm{m}$ thick) through the medial septum of a 13-month-old wild-type female mouse were costained for p75 (green) and ChAT (red). All p $75^{+}$neurons in this region colabel for the cholinergic marker ChAT. Scale bar, $100 \mu \mathrm{m}$.

mals that was not significant when averaged across all slices through the septum. However, when we arranged the slices into groups based on their anteroposterior (rostrocaudal) position, we found a significantly greater proportion of cholinergic neurons in the more posterior (caudal) slices through the medial septum in $p 75^{-/-}$mice (Fig. $7 D$ ). Because $p 75$ is expressed in cholinergic neurons in both anterior and posterior regions, this suggests that developmental cues vary across the basal forebrain resulting in region-specific requirements for p75 in GABAergic development. 
A
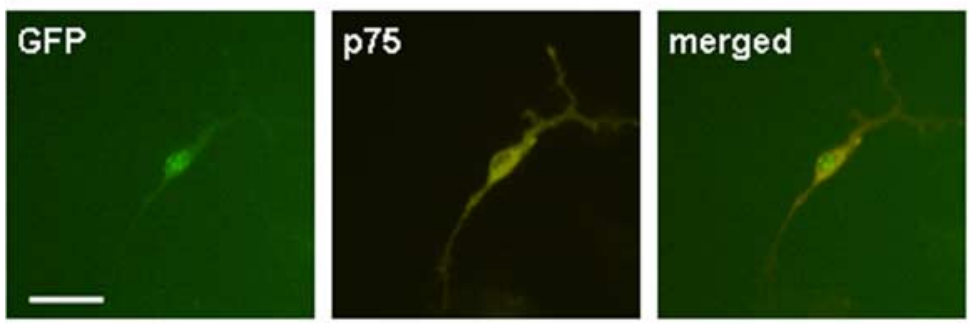

B
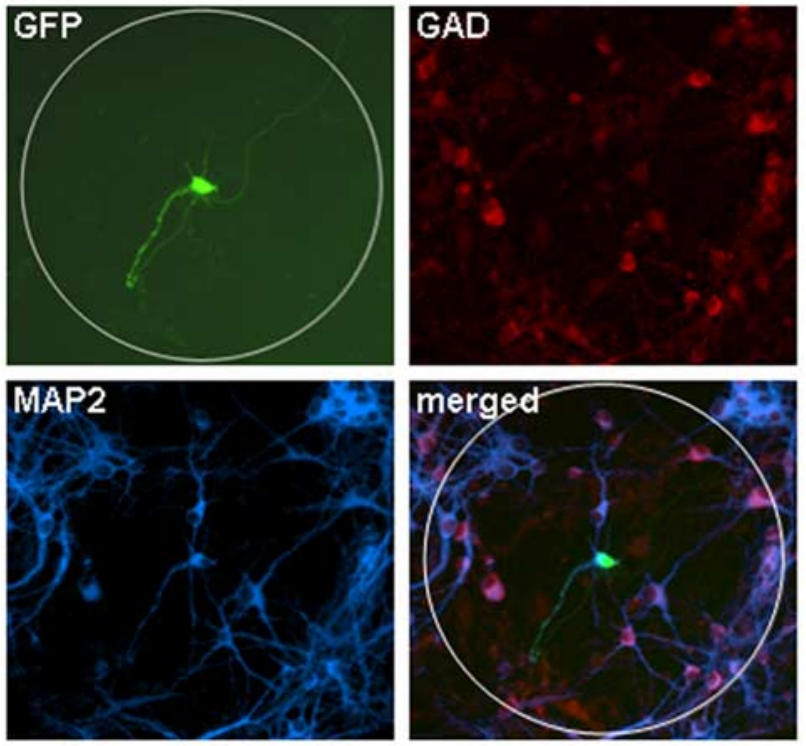

C
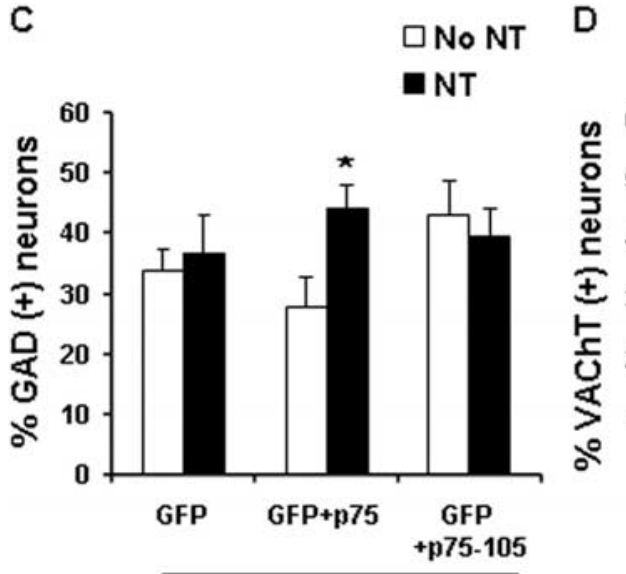

p $75 \%$
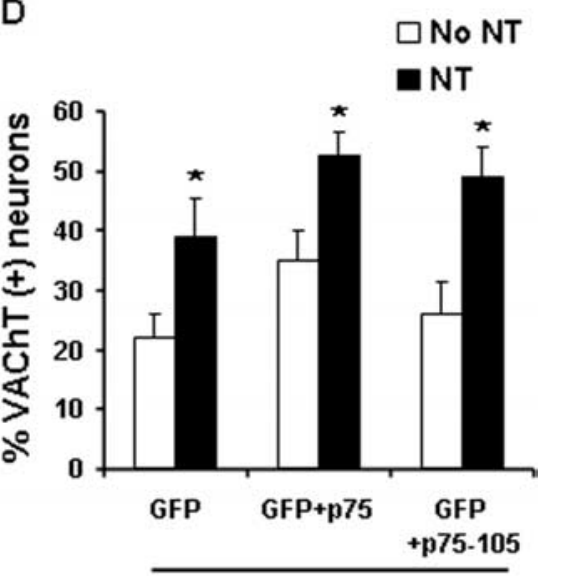

p 75-1-

Figure 5. Exogenous 75 expression modulates neurotrophin responses of surrounding neurons. $A$, Coexpression of GFP (green) and human p75 (yellow) in a basal forebrain neuron derived from a $p 75^{-1-}$ mouse. $B, p 75^{-1-}$ basal forebrain cultures were transfected with GFP (green) and $p 75$ and immunostained for GAD (red) and MAP2 (blue) 6 d later. A 300- $\mu$ m-diameter circle was drawn around the image of an individual $\mathrm{GFP}^{+}$cell, and the numbers of $\mathrm{GAD}^{+}$and $\mathrm{MAP2}{ }^{+}$neurons in the circle were counted. $\boldsymbol{C}$, The percentage of GABAergic neurons in the circle was calculated for neurons transfected with GFP, GFP and human $p 75$, or GFP and the variant human $p 75-105$ and cultured with (NT, filled bars) or without (No NT-open bars) neurotrophins. Values are given as mean \pm SEM percentage of the number of $\mathrm{GAD}^{+}$cells/the number of $\mathrm{MAP}^{+}$cells $(n=6)$. D , The percentage of cholinergic neurons were measured in the surrounding area for neurons transfected with GFP, GFP and human $p 75$, or GFP and the variant human $p 75-105$ (mean \pm SEM; $n=6$ ). ${ }^{*} p<0.05$, unpaired $t$ test.

\section{Discussion}

Local circuits involving GABAergic and cholinergic neurons contribute to the output of basal forebrain projections, contributing to the development and function of cortical circuits.
We have shown that neurotrophins and the p75 receptor regulate the development and relative number of medial septal region basal forebrain cholinergic and GABAergic neurons. Neurotrophins increase the numbers of both neuron types, although only the cholinergic neurons express the p75 neurotrophin receptor. Loss of p 75 does not affect neurotrophin-dependent increases in cholinergic neurons in vitro but limits neurotrophin-dependent development of GABAergic neurons. This non-cellautonomous effect is mediated via release of a soluble factor that promotes the neurotrophin-dependent acquisition of a GABAergic phenotype. Loss of p75 in vivo results in a loss of GABAergic neurons and a region-specific increase in cholinergic neurons. Thus, cell-cell interactions involving neurotrophins and p75 regulate neuronal subpopulations within the medial septum and alter the balance of cholinergic and GABAergic neurons in basal forebrain circuits.

Neurotrophins and GABAergic phenotypes Extensive work in the basal forebrain has shown that neurotrophins induce cholinergic markers (Li et al., 1995; Pongrac and Rylett, 1998; Berse et al., 1999), promote the survival of BFCNs (Hatanaka et al., 1988; Nonomura and Hatanaka, 1992; Nonomura et al., 1995), and increase cholinergic neuron number (Hatanaka et al., 1988; this study). In addition, we found that neurotrophins influence GABAergic development, regulating neuron number and soma size. Thus, neurotrophins regulate the neurotransmitter identity of both major classes of basal forebrain neurons.

New cholinergic or GABAergic neurons could arise by differentiation of a proliferating precursor population, by increased neuronal survival, or by acquisition of phenotypic markers by preexisting neurons. The lack of significant differences in total neuron number or cell division suggests that neurotrophins did not promote the development of new neurons. This is consistent with birthdating studies showing cell cycle withdrawal of basal forebrain neurons during rat embryonic development, with cholinergic neurons born by E17 (Semba and Fibiger, 1988; Brady et al., 1989). Thus, cholinergic and GABAergic increases involve the acquisition or maintenance of phenotypic properties. This is unlikely to reflect a switch from a cholinergic to a GABAergic phenotype because both populations increase after neurotrophin treatment. Rather, neurotrophins may act on noncholinergic, nonGABAergic neurons (Gritti et al., 2006) to promote these neurotransmitter properties.

The lack of change in total neuron number suggests that neu- 
A

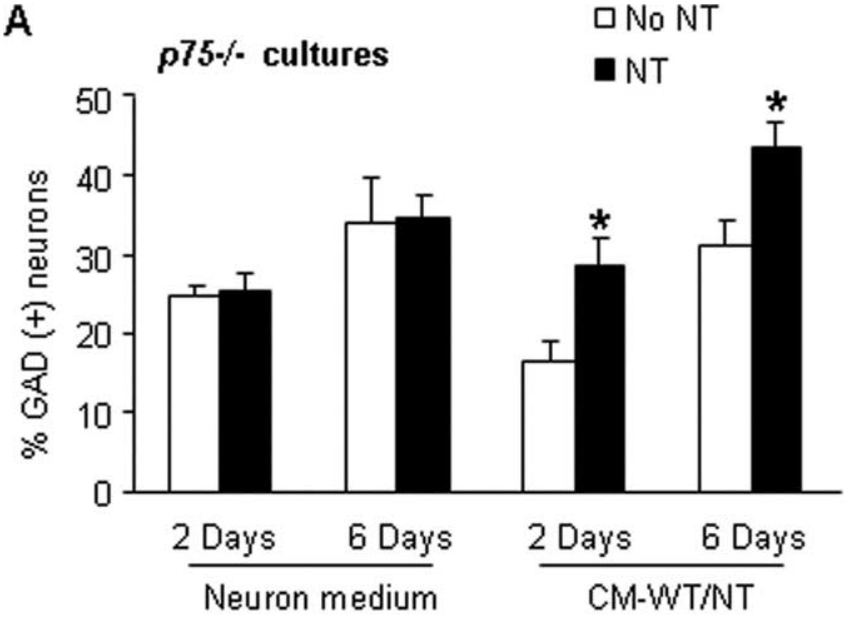

B

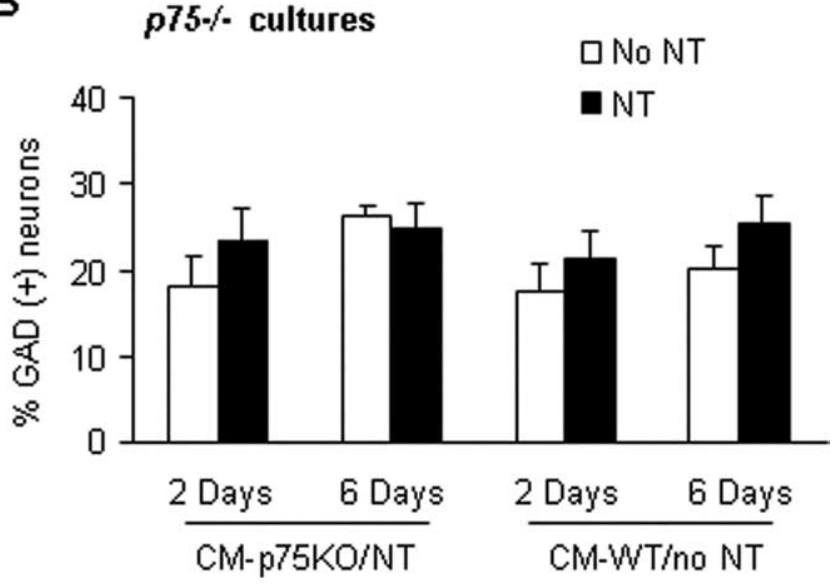

Figure 6. Production of a neurotrophin modulatory activity requires $\mathrm{p} 75$ and neurotrophins. $A, p 75^{-1-}$ basal forebrain neurons were cultured in conditioned medium prepared from neurotrophin-treated wild-type basal forebrain cultures (CM-WT/NT). Neurotrophins (NT, filled bars) induced a significant increase in the percentage of $\mathrm{GAD}^{+}$neurons compared with control (No NT, open bars) in CM-WT/NT-treated, but not untreated, $p 75^{-\prime-}$ cultures. Values are given as mean $\pm \mathrm{SEM}$; neuron medium, $n=4 ; \mathrm{CM}-\mathrm{WT} / \mathrm{NT}, n=5$. $\boldsymbol{B}$, When conditioned medium was made from $p 75^{-1-}$ cultures (CM-p75KO/NT) or from wild-type cultures in the absence of added neurotrophins (CM-WT/no NT), there was no significant change in the percentage of $\mathrm{GAD}^{+}$neurons in $p 75^{-/-}$cultures in response to neurotrophins (mean $\pm \mathrm{SEM} ; n=3$ ). ${ }^{*} p<$ 0.05 , unpaired $t$ test.

rotrophins are not acting as trophic factors for these basal forebrain subpopulations. This fits with studies showing that neurotrophins act as survival factors for mature basal forebrain cholinergic neurons (Burke et al., 1994; Koliatsos et al., 1994) but not young, postnatal neurons (Hatanaka et al., 1988; Nonomura and Hatanaka, 1992). Conversely, several studies show that NGF acts to promote the expression and activity of cholinergic pathway components, including ChAT and VAChT (Hatanaka et al., 1988; Pongrac and Rylett, 1998; Berse et al., 1999), supporting the idea that neurotrophins promote phenotypic properties of cholinergic, and presumably GABAergic, neurons. Expression of cholinergic markers is first seen at approximately E20 in the rat (Bender et al., 1996), several days after the neurons have been born (Semba and Fibiger, 1988). This suggests a period of phenotypic plasticity after the birth of basal forebrain neurons during which the development of cholinergic or GABAergic pheno-
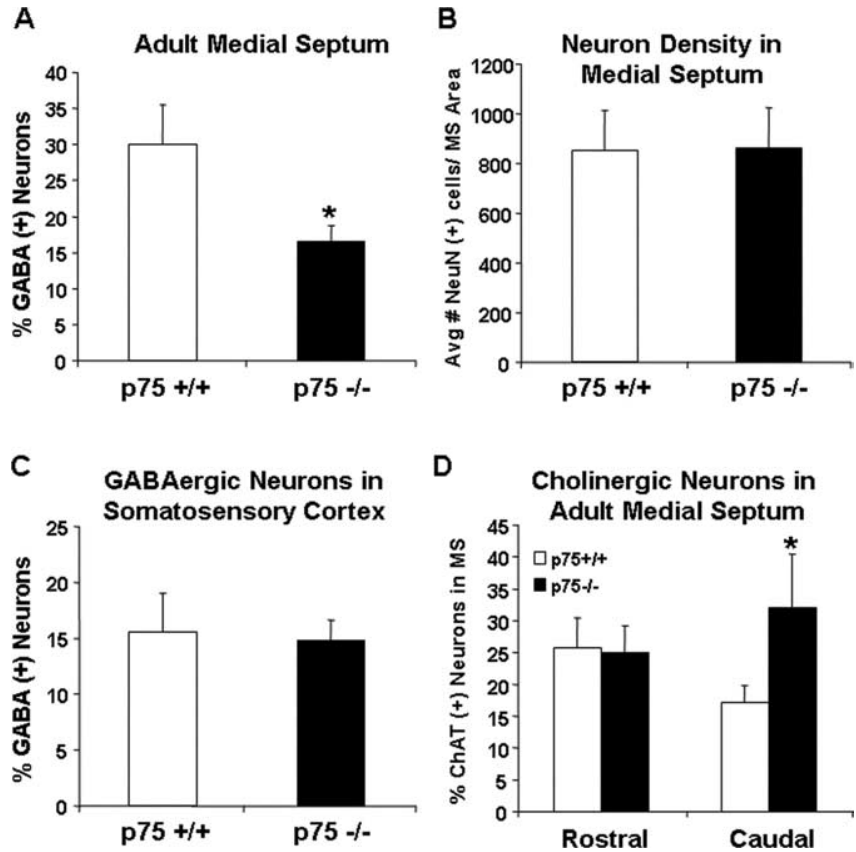

Figure 7. p75 expression is necessary to maintain GABAergic neuron numbers in vivo. Alternating $40 \mu \mathrm{m}$ slices through adult mouse MS from wild-type and $p 75^{-/-}$mice were costained for GABA or ChAT and NeuN. Three-dimensional image series were maximum intensity $z$-projected to generate a composite image. The MS was visually identified and outlined by hand using the NeuN stain image and copied on to the corresponding GABA or ChAT image. $A, G A B A^{+}$ and $\mathrm{NeuN}^{+}$neurons were counted on each image within the defined MS region, and GABAergic neuron percentages were generated. There was a significantly lower percentage of GABA ${ }^{+}$ neurons in adult $p 75^{-1-}$ mice compared with wild types $(n=5$, ranging from 10 to 12 months old; ${ }^{*} p<0.05$, unpaired $t$ test). $\boldsymbol{B}$, There was no difference in the average number of neurons $\left(\mathrm{NeuN}^{+}\right.$cells) in the MS observed between genotypes. $C$, No difference in GABAergic neuron percentages was observed between $p 75^{-/-}$and $p 75^{+/+}$mice in a region of somatosensory cortex $(n=3)$. $\boldsymbol{D}$, The percentages of $\mathrm{ChAT}^{+}$neurons in brain slices through the MS were not significantly different when averaged across all slices. When slices were grouped by their rostral (anterior; $\sim 1.18-1.10 \mathrm{~mm}$ from bregma) and caudal (posterior; $\sim 0.86-0.74 \mathrm{~mm}$ ) position in the brain, we observed a significantly greater percentage of $\mathrm{ChAT}^{+}$neurons in $p 75^{-/-}$mice in the caudal slices ( $n=7$; aged $10-14$ months old; ${ }^{*} p<0.05$, unpaired $t$ test).

types is influenced by the availability of local or target-derived factors.

Our results suggest that postmitotic neurons in the neonatal medial septum retain the potential to develop into either cholinergic or GABAergic neurons. These immature neurons could be committed to the expression of one or the other phenotype or, alternatively, could be plastic in regard to their final neurotransmitter phenotype. Interestingly, this possibility is supported by studies showing that the expression of LIM homeodomain factor L3 (Lhx8, Lhx7) (Mori et al., 2004; Bachy and Retaux, 2006) is necessary for the specification of cholinergic neurons and repression of a GABAergic phenotype in basal forebrain neurons. In fact, suppression of L3 results in GABAergic development in a neuronal cell line that normally differentiates into cholinergic neurons (Manabe et al., 2005).

p75 receptors in the development of basal forebrain neurons Whereas p75 is expressed in BFCNs (Sobreviela et al., 1994), most effects of neurotrophins are mediated via Trk receptors (Knusel et al., 1992; Fagan et al., 1997). p75 has been implicated in the regulation of BFCN number, although analysis of $p 75^{-/-}$mice have produced conflicting data (Van der Zee et al., 1996; Peterson et al., 1997, 1999; Yeo et al., 1997; Ward and Hagg, 1999; Greferath et al., 2000; Naumann et al., 2002). Some differences in pre- 
vious studies could reflect differences in effects of p75 loss along the rostrocaudal axis. We found an increase in the percentage of cholinergic neurons in more caudal regions of the medial septum, with no changes seen in rostral regions. The reasons for these regional differences are not clear but could reflect differences in the timing or availability of trophic factors and the expression of Trk and p75 receptors.

Although p75 limits the number of cholinergic neurons in vivo, we saw no difference in the neurotrophin response of cultured wild-type and $p 75^{-/-}$BFCNs. Because cultures were derived from neonatal animals whereas the number of cholinergic neurons in vivo was analyzed in adult mice, this suggests that there may be developmental changes in p 75 function in the basal forebrain. A number of ligands and interacting proteins regulate p75 function, resulting in discrete effects on cell death, survival, and neuronal function (Dechant and Barde, 2002). Developmental changes in the expression of these $\mathrm{p} 75$-interacting proteins could provide an explanation for the late effects of p75 in limiting cholinergic neuron numbers in vivo.

In contrast to the cholinergic system, the role of p75 in the development of GABAergic basal forebrain neurons has not been addressed. At first glance, this seems an unnecessary question because these neurons do not express p75 (Heckers et al., 1994) (Fig. 3). In culture, we found a complete loss of neurotrophin response in GABAergic neurons derived from the $p 75^{-/-}$mice, suggesting a possible role for p75 in GABAergic development. Interestingly, GABAergic neurons still develop in $p 75^{-/-}$cultures, attaining levels similar to that of neurotrophin-treated wild-type cultures within $6 \mathrm{~d}$. This suggests that p75 may normally act to suppress or delay GABAergic development and that, in vitro, this effect can be overcome by high levels of exogenous neurotrophins.

Examination of the medial septum of $p 75^{-/-}$mice shows that p75 also acts in vivo to regulate the number of GABAergic neurons. Adult $p 75^{-/-}$mice had a smaller percentage of medial septal neurons expressing GABA than strain-matched wild-type animals. Interestingly, we observed this loss in mature adult mice (10-12 months) but not in younger animals ( 3 months; data not shown), suggesting a role for p75 in the maintenance of the GABAergic phenotype in mature animals. The lack of change in younger animals suggests that p75 is not required for the initial establishment of the GABAergic system. This is consistent with our in vitro finding that the number of neonatal GABA neurons is similar in neurotrophin-treated wild-type and $p 75^{-/-}$cultures (at $6 \mathrm{~d}$ ), although the neurotrophin responses and the timing of development are altered. The same mature adult p75-deficient animals that had fewer medial septal GABAergic neurons showed the same or greater percentage of cholinergic neurons. Thus, these data define p75 as a regulator of cholinergic-GABAergic balance in the medial septum.

Non-cell-autonomous mechanisms involving interactions between different cell types have been implicated in neuronal development (Hiramoto et al., 2000; Helmbacher et al., 2003). In the peripheral nervous system, interactions between developing sympathetic neurons and surrounding non-neuronal cells result in the release of a factor that induces TrkA expression in the neurons (Verdi et al., 1996). The lack of p75 expression in wildtype GABAergic neurons and the requirement for p75 for neurotrophin-dependent GABAergic development also suggest a non-cell-autonomous mechanism. Analysis of p75 knock-out neurons and p75 overexpression suggests that p75 in cholinergic neurons is both necessary and sufficient for exogenous neurotrophins to regulate increases in GABAergic neuron number. This non-cell-autonomous expression of p75 results in the release of a soluble factor that influences some GABAergic neurotrophin responses, including the timing of recruitment of new neurons to a GABAergic phenotype. A similar situation is seen in a non-cellautonomous pathway of motor neuron development (Helmbacher et al., 2003). Met, the tyrosine kinase receptor for hepatocyte growth factor, is required for the recruitment of motor neurons to a pea3 (polyoma enhancer activator 3)-positive pool, although it is not expressed in the pea $3^{+}$neurons. Interestingly, met is not required for the initiation of pea 3 expression but for the expansion of the pea $3^{+}$population. Similarly, in the basal forebrain, p75 is required for the neurotrophin-dependent expansion of the GABAergic population. This suggests that $\mathrm{p} 75-$ mediated interactions between cholinergic and GABAergic neurons regulate the ratio of cholinergic to GABAergic neuron number within local basal forebrain circuits, potentially influencing the level of neurotransmission of basal forebrain projections.

\section{References}

Arimatsu Y, Miyamoto M (1991) Survival-promoting effect of NGF on in vitro septohippocampal neurons with cholinergic and GABAergic phenotypes. Brain Res Dev Brain Res 58:189-201.

Bachy I, Retaux S (2006) GABAergic specification in the basal forebrain is controlled by the LIM-hd factor Lhx7. Dev Biol 291:218-226.

Bender R, Plaschke M, Naumann T, Wahle P, Frotscher M (1996) Development of cholinergic and GABAergic neurons in the rat medial septum: different onset of choline acetyltransferase and glutamate decarboxylase mRNA expression. J Comp Neurol 372:204-214.

Berse B, Lopez-Coviella I, Blusztajn JK (1999) Activation of TrkA by nerve growth factor upregulates expression of the cholinergic gene locus but attenuates the response to ciliary neurotrophic growth factor. Biochem J 342:301-308.

Brady DR, Phelps PE, Vaughn JE (1989) Neurogenesis of basal forebrain cholinergic neurons in rat. Brain Res Dev Brain Res 47:81-92.

Brauer K, Seeger G, Hartig W, Rossner S, Poethke R, Kacza J, Schliebs R, Bruckner G, Bigl V (1998) Electron microscopic evidence for a cholinergic innervation of GABAergic parvalbumin-immunoreactive neurons in the rat medial septum. J Neurosci Res 54:248-253.

Burke MA, Mobley WC, Cho J, Wiegand SJ, Lindsay RM, Mufson EJ, Kordower JH (1994) Loss of developing cholinergic basal forebrain neurons following excitotoxic lesions of the hippocampus: rescue by neurotrophins. Exp Neurol 130:178-195.

Dechant G, Barde YA (2002) The neurotrophin receptor p75(NTR): novel functions and implications for diseases of the nervous system. Nat Neurosci 5:1131-1136.

Fagan AM, Garber M, Barbacid M, Silos-Santiago I, Holtzman DM (1997) A role for TrkA during maturation of striatal and basal forebrain cholinergic neurons in vivo. J Neurosci 17:7644-7654.

Freund TF, Meskenaite V (1992) $\gamma$-Aminobutyric acid-containing basal forebrain neurons innervate inhibitory interneurons in the neocortex. Proc Natl Acad Sci USA 89:738-742.

Greferath U, Bennie A, Kourakis A, Bartlett PF, Murphy M, Barrett GL (2000) Enlarged cholinergic forebrain neurons and improved spatial learning in p75 knockout mice. Eur J Neurosci 12:885-893.

Gritti I, Mainville L, Mancia M, Jones BE (1997) GABAergic and other noncholinergic basal forebrain neurons, together with cholinergic neurons, project to the mesocortex and isocortex in the rat. J Comp Neurol 383:163-177.

Gritti I, Henny P, Galloni F, Mainville L, Mariotti M, Jones BE (2006) Stereological estimates of the basal forebrain cell population in the rat, including neurons containing choline acetyltransferase, glutamic acid decarboxylase or phosphate-activated glutaminase and colocalizing vesicular glutamate transporters. Neuroscience 143:1051-1064.

Ha DH, Robertson RT, Roshanaei M, Weiss JH (1999) Enhanced survival and morphological features of basal forebrain cholinergic neurons in vitro: role of neurotrophins and other potential cortically derived cholinergic trophic factors. J Comp Neurol 406:156-170.

Hagg T, Van der Zee CEEM, Ross GM, Riopelle RJ (1997) Basal forebrain neuronal loss in mice lacking neurotrophin receptor p75. Science 277:838-839. 
Hartikka J, Hefti F (1988) Development of septal cholinergic neurons in culture: plating density and glial cells modulate effects of NGF on survival, fiber growth, and expression of transmitter-specific enzymes. J Neurosci 8:2967-2985.

Hatanaka H, Tsukui H, Nihonmatsu I (1988) Developmental change in the nerve growth factor action from induction of choline acetyltransferase to promotion of cell survival in cultured basal forebrain cholinergic neurons from postnatal rats. Brain Res 467:85-95.

Heckers S, Ohtake T, Wiley RG, Lappi DA, Geula C, Mesulam MM (1994) Complete and selective cholinergic denervation of rat neocortex and hippocampus but not amygdala by an immunotoxin against the p75 NGF receptor. J Neurosci 14:1271-1289.

Helmbacher F, Dessaud E, Arber S, deLapeyriere O, Henderson CE, Klein R, Maina F (2003) Met signaling is required for recruitment of motor neurons to PEA3-positive motor pools. Neuron 39:767-777.

Hiramoto M, Hiromi Y, Giniger E, Hotta Y (2000) The Drosophila Netrin receptor Frazzled guides axons by controlling Netrin distribution. Nature 406:886-889.

Hohmann CF (2003) A morphogenetic role for acetylcholine in mouse cerebral neocortex. Neurosci Biobehav Rev 27:351-363.

Knusel B, Rabin S, Widmer HR, Hefti F, Kaplan DR (1992) Neurotrophininduced trk receptor phosphorylation and cholinergic neuron response in primary cultures of embryonic rat brain neurons. NeuroReport 3:885-888.

Koliatsos VE, Price DL, Gouras GK, Cayouette MH, Burton LE, Winslow JW (1994) Highly selective effects of nerve growth factor, brain-derived neurotrophic factor, and neurotrophin-3 on intact and injured basal forebrain magnocellular neurons. J Comp Neurol 343:247-262.

Kordower JH, Chen EY, Sladek Jr JR, Mufson EJ (1994) trkimmunoreactivity in the monkey central nervous system: forebrain. J Comp Neurol 349:20-35.

Lee KF, Li E, Huber LJ, Landis SC, Sharpe AH, Chao MV, Jaenisch R (1992) Targeted mutation of the gene encoding the low affinity NGF receptor p75 leads to deficits in the peripheral sensory nervous system. Cell 69:737-749.

Li Y, Holtzman DM, Kromer LF, Kaplan DR, Chua-Couzens J, Clary DO, Knusel B, Mobley WC (1995) Regulation of TrkA and ChAT expression in developing rat basal forebrain: evidence that both exogenous and endogenous NGF regulate differentiation of cholinergic neurons. J Neurosci 15:2888-2905.

Lopez-Coviella I, Follettie MT, Mellott TJ, Kovacheva VP, Slack BE, Diesl V, Berse B, Thies RS, Blusztajn JK (2005) Bone morphogenetic protein 9 induces the transcriptome of basal forebrain cholinergic neurons. Proc Natl Acad Sci USA 102:6984-6989.

Manabe T, Tatsumi K, Inoue M, Matsuyoshi H, Makinodan M, Yokoyama S, Wanaka A (2005) L3/Lhx8 is involved in the determination of cholinergic or GABAergic cell fate. J Neurochem 94:723-730.

Mori T, Yuxing Z, Takaki H, Takeuchi M, Iseki K, Hagino S, Kitanaka J, Takemura M, Misawa H, Ikawa M, Okabe M, Wanaka A (2004) The LIM homeobox gene, L3/Lhx8, is necessary for proper development of basal forebrain cholinergic neurons. Eur J Neurosci 19:3129-3141.

Naumann T, Casademunt E, Hollerbach E, Hofmann J, Dechant G, Frotscher M, Barde YA (2002) Complete deletion of the neurotrophin receptor p75NTR leads to long-lasting increases in the number of basal forebrain cholinergic neurons. J Neurosci 22:2409-2418.

Nishimura A, Hohmann CF, Johnston MV, Blue ME (2002) Neonatal electrolytic lesions of the basal forebrain stunt plasticity in mouse barrel field cortex. Int J Dev Neurosci 20:481-489.
Nonomura T, Hatanaka H (1992) Neurotrophic effect of brain-derived neurotrophic factor on basal forebrain cholinergic neurons in culture from postnatal rats. Neurosci Res 14:226-233.

Nonomura T, Nishio C, Lindsay RM, Hatanaka H (1995) Cultured basal forebrain cholinergic neurons from postnatal rats show both overlapping and non-overlapping responses to the neurotrophins. Brain Res 683:129-139.

Paxinos G, Franklin KBJ (2001) The mouse brain atlas in stereotaxic coordinates, Ed 2. San Diego: Academic.

Peterson DA, Leppert JT, Lee KF, Gage FH (1997) Basal forebrain neuronal loss in mice lacking neurotrophin receptor p75. Science 277:837-839.

Peterson DA, Dickinson-Anson HA, Leppert JT, Lee KF, Gage FH (1999) Central neuronal loss and behavioral impairment in mice lacking neurotrophin receptor p75. J Comp Neurol 404:1-20.

Plaschke M, Naumann T, Kasper E, Bender R, Frotscher M (1997) Development of cholinergic and GABAergic neurons in the rat medial septum: effect of target removal in early postnatal development. J Comp Neurol 379:467-481.

Pongrac JL, Rylett RJ (1998) NGF-induction of the expression of ChAT mRNA in PC12 cells and primary cultures of embryonic rat basal forebrain. Brain Res Mol Brain Res 62:25-34.

Rudge JS, Eaton MJ, Mather P, Lindsay RM, Whittemore SR (1996) CNTF induces raphe neuronal precursors to switch from a serotonergic to a cholinergic phenotype in vitro. Mol Cell Neurosci 7:204-221.

Semba K (2000) Multiple output pathways of the basal forebrain: organization, chemical heterogeneity, and roles in vigilance. Behav Brain Res 115:117-141.

Semba K, Fibiger HC (1988) Time of origin of cholinergic neurons in the rat basal forebrain. J Comp Neurol 269:87-95.

Sobreviela T, Clary DO, Reichardt LF, Brandabur MM, Kordower JH, Mufson EJ (1994) TrkA-immunoreactive profiles in the central nervous system: colocalization with neurons containing p75 nerve growth factor receptor, choline acetyltransferase, and serotonin. J Comp Neurol 350:587-611.

Van der Zee CE, Ross GM, Riopelle RJ, Hagg T (1996) Survival of cholinergic forebrain neurons in developing p75NGFR-deficient mice. Science 274:1729-1732.

Verdi JM, Groves AK, Farinas I, Jones K, Marchionni MA, Reichardt LF, Anderson DJ (1996) A reciprocal cell-cell interaction mediated by NT-3 and neuregulins controls the early survival and development of sympathetic neuroblasts. Neuron 16:515-527.

Ward NL, Hagg T (1999) p75(NGFR) and cholinergic neurons in the developing forebrain: a re-examination. Brain Res Dev Brain Res 118:79-91.

Ward NL, Hagg T (2000) BDNF is needed for postnatal maturation of basal forebrain and neostriatum cholinergic neurons in vivo. Exp Neurol 162:297-310.

Yan H, Chao MV (1991) Disruption of cysteine-rich repeats of the p75 nerve growth factor receptor leads to loss of ligand binding. J Biol Chem 266:12099-12104.

Yang B, Slonimsky JD, Birren SJ (2002) A rapid switch in sympathetic neurotransmitter release properties mediated by the p 75 receptor. Nat Neurosci 5:539-545.

Yeo TT, Chua-Couzens J, Butcher LL, Bredesen DE, Cooper JD, Valletta JS, Mobley WC, Longo FM (1997) Absence of p75NTR causes increased basal forebrain cholinergic neuron size, choline acetyltransferase activity, and target innervation. J Neurosci 17:7594-7605.

Zaborszky L, Duque A (2000) Local synaptic connections of basal forebrain neurons. Behav Brain Res 115:143-158. 